\title{
Three-channel laser diode driver for multimedia laser projectors
}

\author{
Svetozar Ilchev, Rumen Andreev, Zlatoliliya Ilcheva, Ekaterina Otsetova-Dudin \\ Received: June 17, 2020, Revised: July 24, 2020. Accepted: August 10, 2020. Published: August 13, 2020.
}

\begin{abstract}
The paper describes the design, implementation and test results of a three-channel laser diode driver intended for use in multimedia laser projectors. Our development goals were to create a compact and power-efficient driver, which achieves good color mixing and supports high modulation frequencies. It is compatible with most laser diode configurations used in multimedia laser projectors with a total optical output power of several watts. The driver is equipped with three protected signal modulation inputs. It needs a single regulated power supply between $9 \mathrm{~V}$ and $18 \mathrm{~V}$ and controls up to three cooling fans intended for the thermal management of the laser diodes. Our initial tests show that the driver works very well and is suitable for the long-term operation in projector systems. In the future, we plan to perform more tests on the signal feedback for various input signal combinations, which should result in optimizations of the feedback configuration and improvements of the modulation response of the driver.
\end{abstract}

Keywords — Laser diode driver, Multimedia laser projector, Laser animations, Embedded systems.

\section{INTRODUCTION}

$\mathrm{S}_{\mathrm{fo}}^{\mathrm{E}}$ EMICONDUCTOR light sources are a modern solution for providing illumination in both everyday life and specialized applications in the industry, agriculture or multimedia entertainment. Compared to traditional light sources such as halogen lamps, they have multiple advantages, e.g. high-efficiency, small dimensions and long life. Among their disadvantages are the relatively high cost and the need for electronic regulation of their power supply. Laser diodes are semiconductor laser light sources that emit coherent light with a specific wavelength that may range from ultraviolet $(<$ $400 \mathrm{~nm})$ to infrared $(>750 \mathrm{~nm})$. They are used in multimedia laser projectors to draw laser animations on a surface, in

This research is supported by the National Scientific Program "Information and Communication Technologies for a Single Digital Market in Science, Education and Security (ICTinSES)", financed by the Ministry of Education and Science.

$\mathrm{S}$. Ilchev is with the Institute of Information and Communication Technologies, Bulgarian Academy of Sciences, Acad. G. Bonchev str. 2, 1113 Sofia, Bulgaria (phone: +359 2 9796611, fax: +359 2 8707273, e-mail: svetozar@ilchev.net).

R. Andreev is with the Institute of Information and Communication Technologies, Bulgarian Academy of Sciences, Acad. G. Bonchev str. 2, 1113 Sofia, Bulgaria (e-mail: rumen@isdip.bas.bg).

$Z$. Ilcheva is with the Institute of Information and Communication Technologies, Bulgarian Academy of Sciences, Acad. G. Bonchev str. 2, 1113 Sofia, Bulgaria (e-mail: zlat@isdip.bas.bg).

E. Otsetova-Dudin is with the University of Telecommunications and Posts, Acad. Stefan Mladenov str. 1, 1700 Sofia, Bulgaria (e-mail: eotsetova@abv.bg). industrial positioning lasers to project alignment markings, in the backlight illumination of computer DLP projectors, etc.

When a product that contains laser diodes is designed, several important aspects must be taken into consideration. First, an electronic driver must control the current through the laser diode to maintain the desired level of brightness and prevent the diode from burning out. Second, the physical dimensions of laser diodes - usually cylindrical shapes with a diameter of less than $9 \mathrm{~mm}$ - are insufficient to ensure proper cooling, so they must be mounted in a suitable copper, brass or aluminum housing that has contact to a heatsink or a thermoelectric cooling element. In addition, cooling fans are employed to create airflow and reduce the temperature inside the product enclosure.

In this paper, an electronic driver for laser diodes is proposed that is specifically developed for multimedia laser projectors. These projectors generally employ strings of one or more diodes of three different colors: red $(635-640 \mathrm{~nm})$, green $(520-532 \mathrm{~nm})$ and blue $(440-450 \mathrm{~nm})$. When combined, they form a white beam with a total optical output power of several watts. The laser beam is deflected by two rapidly moving mirrors within the projector enclosure, so an animation may be projected on a remote surface. The speed of the mirrors is usually between 20 and $40 \mathrm{kpps}$ (kilo points per second) at scanning angles up to $8^{\circ}$.

As a result, several important requirements for the laser diode driver are identified. First, the driver should be capable of driving three independent strings of one or more laser diodes. The intensity of each string should be modulated independently of the other strings. Second, the modulation speed of the intensity of each color channel should be faster than $40 \mathrm{kHz}$, preferably around $100 \mathrm{kHz}$. Third, as the power supply of all laser diodes in the projector passes through the laser diode driver, it should be designed in a very powerefficient manner. The power efficiency leads to a reduction of the wasted power in the form of heat, which makes possible the use of smaller heatsinks. As most multimedia laser projectors are designed as portable units, their dimensions and weight are important. As a consequence, both the heatsink and the laser driver should be as small as possible.

In summary, our aim during the development of the proposed laser diode driver was to achieve an accurate and sufficiently fast modulation $(>40 \mathrm{kHz})$ of three independent color channels along with a high-quality white beam at full output power, while minimizing the amount of generated heat and reducing the physical dimensions of the driver as much as 
possible.

The next section of the paper presents some related work. Then, the design of the laser driver is described on both a conceptual and an implementation level. Finally, some experimental results are presented and the paper is concluded with some directions for future work and improvements.

\section{RELATED WORK}

Some work related to laser diodes and the required electronic drivers is presented in the following paragraphs.

The authors of [1] present the combination of electronic drivers and multiple infrared laser diodes on the same board. The dimensions are made as small as possible. In [2], a laser diode driver is proposed that has a current control based on two fixed voltage references. The author of [3] and [4] has designed several laser diode drivers. In [3], a laser diode driver for $3 \mathrm{D}$ scanning is proposed, which is capable of providing up to $35 \mathrm{~A}$ of current through the laser diode. Another powerful laser diode driver is presented in [4]. It supplies up to $150 \mathrm{~A}$ of current. For this purpose, it uses new developments in the field of FET transistors based on Gallium nitride $(\mathrm{GaN})$ and provides a total input power to the laser diode string of up to $4 \mathrm{~kW}$. In [5], a highly efficient laser diode driver is presented. It has small dimensions and excellent thermal dissipation capabilities.

In the study presented in [6], a fast laser diode driver is proposed for the power supply of laser diodes with currents up to $80 \mathrm{~mA}$. Its goal is to deliver very short pulses (around $2 \mathrm{~ns}$ ) at rates of up to $40 \mathrm{MHz}$. In [7], a laser diode driver is proposed that integrates a dedicated power converter to improve the efficiency. The supported maximum current of about $2.5 \mathrm{~A}$ is enough to power many popular laser diodes used in multimedia laser projectors. The authors of [8] propose a laser diode driver for a system for imaging applications with fast current modulation. It allows modulation pulses of less than $10 \mathrm{~ns}$ at repetition rates of more than $500 \mathrm{kHz}$.

From an application point of view, laser diodes target multiple application fields and extend the applications associated with traditional solid-state or gas lasers (e.g. [9]). Some innovative applications of laser systems include the replacement of traditional touchscreens by laser-based sensor modules, which contain laser emitters and laser light sensors as described in [10]. Interesting multimedia effects may be created by a combination of laser beams and Tesla coils [11]. The laser beams may guide the Tesla coil discharges to create electrical discharges that are up to $1.1 \mathrm{~m}$ in length. An important field of applications of laser systems is industrial engraving, cutting and drilling or analysis of materials (see e.g. $[12,13,14]$ ), where semiconductor laser light sources benefit from high-speed analog modulation.

Possible application fields for the proposed driver include laser projectors for interactive multimedia education $[15,16]$, information screens for transportation networks [17] or for data from wireless sensor networks $[18,19,20]$, head-up displays for sensor data from devices for robot-assisted surgeries [21], etc.

Our paper differs from the cited papers that are part of the current state of the art by proposing a driver that is optimized with regard to several criteria at once, all of which are of major importance for multimedia laser projectors. These criteria include the presence of responsive and accurate modulation inputs, good white color quality at maximum power, efficient energy conversion, small size and easy installation, presence of fan control circuits, options for emergency shutdown and status indication, and, last but not least, acceptable production price.

From an application point of view, the benefits of our driver consist in the achievement of a well-thought-out combination between the aforementioned criteria, which will make it an excellent laser driver board for medium-power laser projectors in the power range of $3 \mathrm{~W}$ to $8 \mathrm{~W}$.

\section{CONCEPTUAL Design}

In order to design the building blocks of the driver, we have to analyze carefully the requirements and the criteria that are briefly outlined in the previous sections. As part of this initial analysis, we can identify two major design aspects that are important.

The first aspect is related to making a decision on how the power that comes from the power supply will be transformed before it reaches the laser diode strings. The possible choices that can be made include passing the power directly through a resistor or using a suitable integrated circuit - either a linear regulator or a switching regulator. In order to achieve good efficiency, reduce the board size, and allow flexibility in the choice of power supplies and laser diode strings, we decided to use a switching regulator. The disadvantage of this decision is that it raises the complexity of the board and the production cost.

The second aspect is related to making a decision on how the input signal modulation will control the current through the laser diodes. One possible solution is to use a switching regulator that has some integrated form of control input designed to switch on/off or limit either its output voltage or its output current. Another approach is to use a dedicated form of control implemented through discrete integrated circuits and transistors. The first solution is simple and elegant and minimizes the cost and the size of the board and most switching regulators have a control input pin. The major disadvantages of this solution are that most control inputs are digital and that the modulation speeds are usually limited to several kilohertz, which is insufficient for our purposes.

A specific idea that we explored at that stage was to use a dedicated integrated circuit for driving light-emitting diodes (LEDs) such as LED2001 from ST. It provides good efficiency and works well for constant currents or for digital input signal modulation up to about $2 \mathrm{kHz}$ [22]. Unfortunately, it is unsuitable for input signal modulation speeds of around $20-40 \mathrm{kHz}$ that are needed for multimedia laser projectors. 
Another disadvantage is that the high-quality color mixing we want to achieve requires either an analog input modulation or very fine-grained digital modulation to ensure the good quality of the output white beam.

For the reasons above, we chose the second approach and decided to combine the use of a switching regulator with an analog input signal modulation in the range $0-5 \mathrm{~V}$ implemented through discrete integrated circuits and highspeed transistors. The disadvantage is that this approach makes the board bigger, more complex and raises the production cost.

At this stage, let us illustrate why it is important to use a combination of a switching regulator and transistor-based input modulation and how it influences the efficiency of the driver and reduces the heat dissipation.

First, let us do a calculation of the dissipated power without a switching regulator. If a $12 \mathrm{~V}$ power supply is used to provide $1.5 \mathrm{~A}$ of current to one Nichia NDG7475 laser diode with a forward voltage $V_{f}$ of $4.6 \mathrm{~V}$, then the dissipated heat in the main transistor corresponds to the following calculation: $P_{D H T}=12 \mathrm{~V} \times 1.5 \mathrm{~A}-4.6 \mathrm{~V} \times 1.5 \mathrm{~A}-R_{s} \times 1.5 \mathrm{~A}^{2}=18 \mathrm{~W}-$ $6.9 \mathrm{~W}-0.225 \mathrm{~W}=10.875 \mathrm{~W}$, where $P_{D H T}$ is the dissipated heat in the transistor and $R_{s}$ is the value in ohms (usually around $0.1 \Omega$ ) of the resistor used to sense the current as part of the feedback circuit for current control. This is a very large loss of power for a single-color green beam with an optical output power of about $1 \mathrm{~W}$. The solution is to match the power supply voltage to the total forward voltage of the connected laser diode string. As is typical for most semiconductor diodes, laser diodes are not suited for connection in parallel. Instead, they are connected in series, so a higher power supply voltage is needed when more than one laser diode is used. The number of laser diodes is different for the different color channels due to differences in their optical output power and the biological characteristics of the human eye, which is most sensitive to green color. A typical combination includes multiple red diodes, several blue diodes and one or two green diodes. Thus, a power supply of $15 \mathrm{~V}-24 \mathrm{~V}$ is usually needed for the red and blue diodes, while the green diodes may be powered by a $12 \mathrm{~V}$ power supply. In this case, either two separate power supplies of different output voltage have to be used, or the laser diode driver should be equipped with one or more integrated circuits for power supply conversion. In order to use a single nonadjustable power supply and to achieve a reduction of the dimensions and the price of the multimedia laser projector, we equip the laser diode driver with a DC-DC converter that lowers the power supply voltage before passing it to the transistor-based current regulation for each color channel. The power conversion efficiency is typically around $85-90 \%$, which results in a significant reduction of the heat dissipation. In the usual case $P_{D H C}=(6.9 \mathrm{~W}+0.225 \mathrm{~W}) \times 15 \% \approx 1 \mathrm{~W}$, where $P_{D H C}$ is the heat dissipated in the DC-DC converter, and $P_{D H T} \approx 0.1 \mathrm{~W}$ heat dissipation for the transistor, if we use a MOSFET transistor with a small enough on-resistance. In practice, some voltage tolerance of $0.5 \mathrm{~V}-1 \mathrm{~V}$ should be left to be compensated by the transistor due to fluctuations in the forward voltage of the laser diodes, so the realistic maximum dissipated power in the transistor $P_{D H T}$ is $1-2 \mathrm{~W}$.

As the driver regulates the current of three different color channels, three different power supply voltages are needed (or at least two voltages if the blue and red power supply voltages are combined). To minimize the complexity of the driver and achieve further reduction in its dimensions, we chose the TPS65261 integrated circuit from Texas Instruments. The integrated circuit is a step-down (buck) DC-DC converter, which provides three separate power supply channels, whose voltage can be adjusted individually. The first output channel delivers up to $3 \mathrm{~A}$ of current and the other two channels deliver up to $2 \mathrm{~A}$ of current. One advantage of using this converter consists in the possibility for reduction of ripples in the input current by using different clocks for the different output channels that are out-of-phase in relation to each other. Another advantage is the reduction of the total number of components needed for the generation of the power supply for each channel, as only one integrated circuit that is common for all channels is used. This is beneficial because it reduces both the complexity of the driver and its physical dimensions. In addition, the run-time status of the driver can be communicated to an external microcontroller with fewer components as the "power-good" output of the step-down converter summarizes the status of all output channels. The emergency shutdown of the laser diode driver is also facilitated, as only one integrated circuit has to be enabled or disabled by the external emergency shutdown signal (Fig. 2).

Last but not least, for the effective temperature regulation, the driver includes independent control circuits for up to three fans. The fan activity is controlled by thermistors, so that if the temperature is below a predefined value, the fans are turned off. We decided to include these fan control circuits as part of the laser diode driver because the other electronic boards in a multimedia laser projector may not be capable of controlling the fans and we wanted to reduce the acoustic emissions of the laser projector and prolong the fan lifespan.

Fig. 1 shows the functional blocks of the laser diode driver (100) that we designed as a result of the requirements analysis. The power supply block (110) contains a fuse (111) for overcurrent protection, a TVS-diode (112) for overvoltage protection, a MOSFET transistor (113) for polarity reversal protection and an electrolytic bulk capacitor (114) that functions as an energy buffer for the driver. The three-channel DC-DC converter (115) reduces the power supply voltage to the respective values that are necessary for the specific laser diode configuration chosen for the laser projector. The emergency shutdown circuit (116) provides an external input for shutting down the power to the laser diodes in case of emergency, e.g. high operating temperature, human-related accidents, etc. This input is designed to be activated from a microcontroller unit and accepts voltage levels of up to $5 \mathrm{~V}$. The status output (117) indicates the operational status of the DC-DC converter (115). In case of an error, it is set to $0 \mathrm{~V}$.

For each color channel, the DC-DC converter (115) requires some dedicated components that are part of the 
voltage regulation block (120). First, an input capacitor (121), several output capacitors (122) and an inductor (123) provide input and output energy storage for the specific channel. There is also a bootstrap capacitor (124) for biasing the highside MOSFET transistor integrated into the DC-DC converter (115). The voltage divider (125) sets the output voltage for the channel and the compensation circuit (126) improves the stability of the regulation by preventing oscillations. The anode of the laser diode (127) is connected to the output voltage and the cathode is connected to the drain of the MOSFET transistor (135) of the corresponding current regulation block (130).

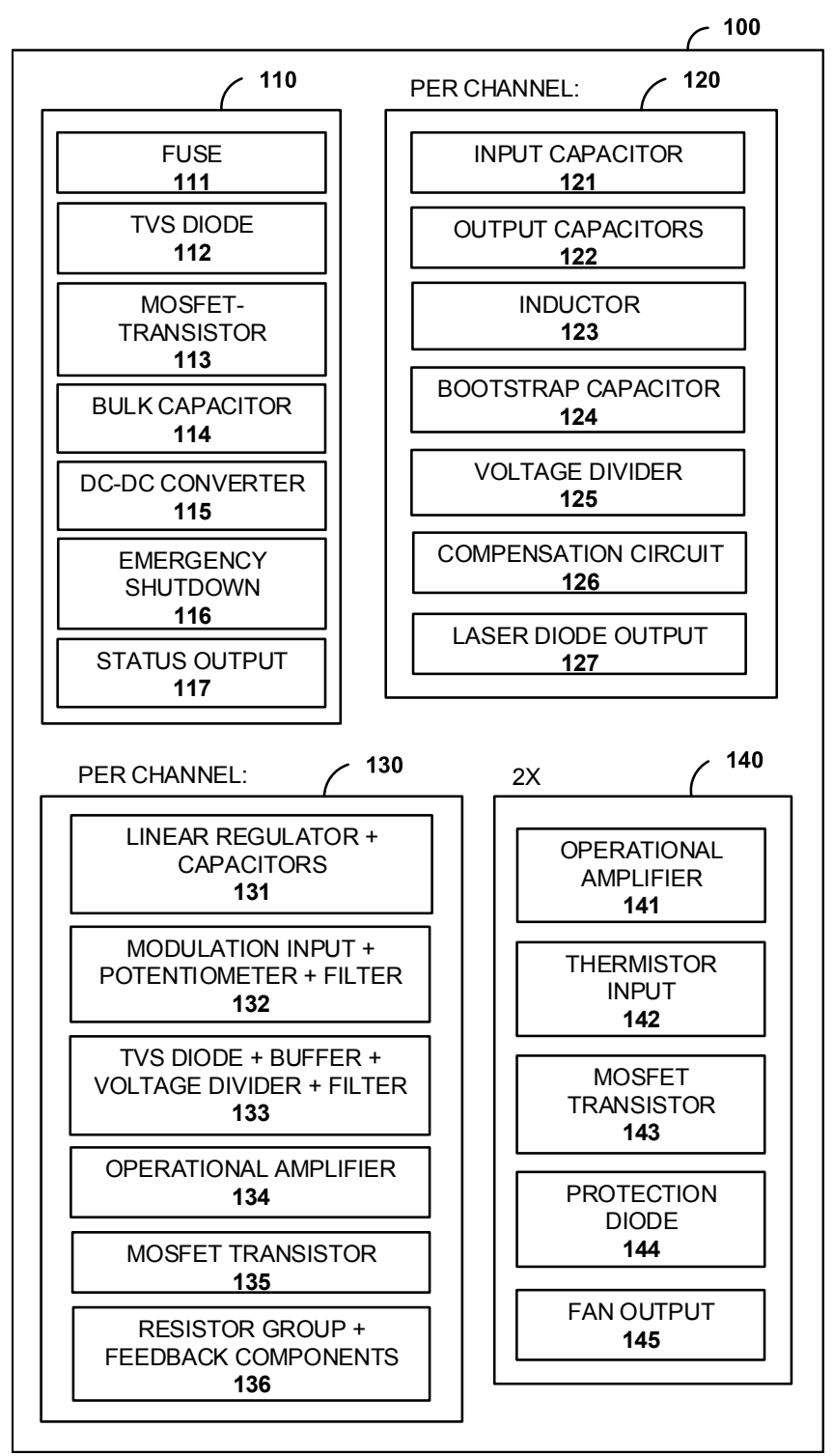

Fig. 1 Functional blocks of the laser diode driver

For each color channel, there is a separate current regulation block (130), which contains several functional groups of components. First, a linear regulator equipped with an input and an output capacitor (131) provides a power supply voltage of $5 \mathrm{~V}$ to the other components in the block.
The analog modulation input, a potentiometer and a low-pass filter circuit (132) receive, scale and filter the input signal that controls the current through the laser diode (127). The potentiometer is mounted on the control panel of the laser projector and enables the end user of the laser projector to change the current and hence the brightness of each color channel individually. Then, a buffer (133) implemented through an operational amplifier, improves the input signal impedance. At the input of the buffer, a TVS protection diode is connected to clamp any potential overvoltage. At the output of the buffer, a voltage divider and a low-pass filter adjust and filter the signal value. The voltage divider sets the maximum current limit for the laser diode string. Then, the signal is passed to the positive input of the operational amplifier (134) that performs the actual current regulation. The negative input of the operational amplifier (134) is connected to the current sensing resistor group (136), which also contains several feedback components that improve the stability of the current regulation and reduce the probability for current overshooting and oscillations. The output of the operational amplifier (134) is connected to the gate of the N-channel MOSFET transistor (135) that controls the actual current through the laser diode. The source of the MOSFET transistor (135) is connected to the current sensing resistor group (136). The other end of the resistor group is connected to the ground of the driver board.

There are two identical functional blocks for fan control (140). Each of them contains an operational amplifier (141), which controls the gate of the N-channel MOSFET transistor (143). The transistor switches on/off the fan connected to the fan output (145). The thermistor input (142) is intended for one $10 \mathrm{k} \Omega$ thermistor that is connected to the ground of the laser driver board and to the negative input of the operational amplifier (141). At around $50^{\circ} \mathrm{C}$ its resistance is small enough to turn high the output of the operational amplifier (141), which switches on the MOSFET transistor (143) and the fan. The protection diode (144) is a freewheeling diode that protects the transistor from overvoltage when the fan is switched off.

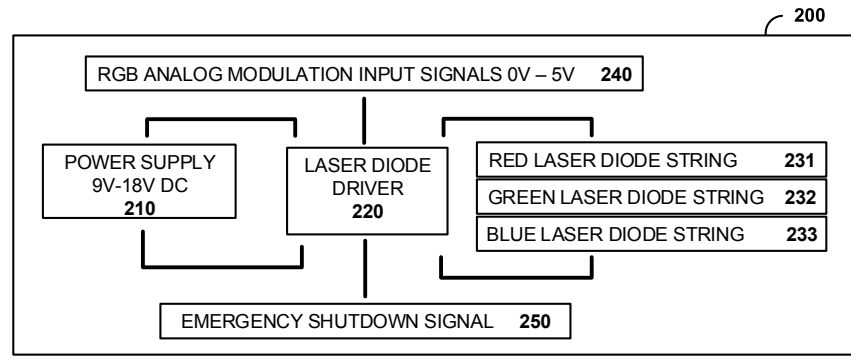

Fig. 2 Use of the laser diode driver in a multimedia laser projector

Fig. 2 shows the integration of the laser diode driver (220) in a multimedia laser projector (200). A 9V-18V DC power supply (210) is connected to the driver (220), which controls the current through the red (231), green (232) and blue (233) laser diode strings. The driver accepts red, green and blue (RGB) analog modulation input signals (240) in the range $0 \mathrm{~V}$ - 
$5 \mathrm{~V}$ from an external board, e.g. an ILDA signal distribution board. An emergency shutdown signal (250) from an external microcontroller unit can switch off the laser diodes in case of emergencies by disabling the DC-DC converter (115).

\section{DRIVER IMPLEMENTATION}

The implementation of the driver has been done in the form of a printed circuit board (PCB). The PCB was designed by means of the open source software KiCad. Fig. 3 shows the PCB schematics that pertain to the DC-DC converter (115) and the provision of power supply voltage to the three laser diode channels. The schematics of the power supply block (110), the voltage regulation block (120), the fan control block (140) and an experimental fan control circuit are also presented.

The finished board is shown in Fig. 4. Some of the important functional blocks and groups from Fig. 1 are marked by means of arrows. We chose a two-layer board setup and we used predominantly surface-mount (SMD) components that are placed exclusively on the top layer of the board. The three current regulation MOSFET transistors in TO-220 housings (135) that are placed along the right edge of the board (Fig. 4) are among the few classic through-hole components. They are mounted vertically to facilitate their attachment to a heatsink as they dissipate some heat during operation.
We decided to implement each voltage divider (133) as a 25-turn trimmer located to the left of the MOSFET transistor (135) for the corresponding color channel (Fig. 4). The trimmers enable the designer of the laser projector to adjust with high accuracy the maximum current for each diode string during the color calibration phase. The maximum current determines the maximum intensity of each color channel and, in this way, we can set the proper color mixture for obtaining a good-quality white color out of the specific laser diodes used in the projector. Thus, the voltage divider (133) is a necessary element for achieving our development aims.

For the purpose of easier color calibration, we also added a constant current jumper for each color channel. The jumpers can be seen set in position in Fig. 4 - next to the connectors for analog input modulation signals (132).

If the jumper for a specific color channel is set, a voltage of $5 \mathrm{~V}$ is applied at the modulation input of the same channel (132). This voltage is taken from the output of the linear regulator (131) located at the top part of the board (Fig. 4). In this case, the maximum current value set by the voltage divider (133) is passed through the corresponding laser diode string. The constant current jumper has two important advantages during the setup of the laser projector. First, no external system generating modulation signals needs to be connected to the projector. This saves time during the initial setup and reduces the number of wires that have to be handled

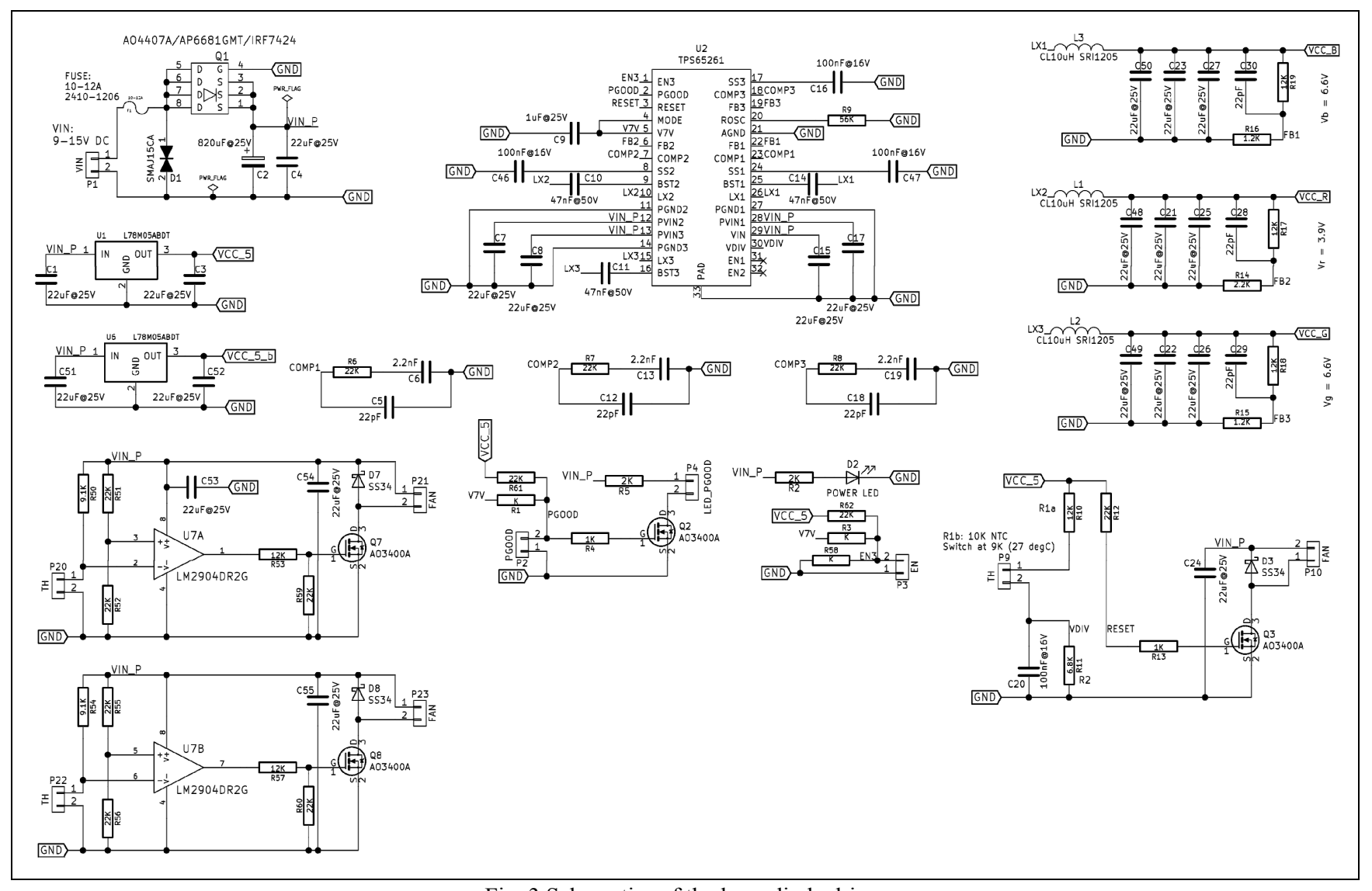

Fig. 3 Schematics of the laser diode driver 
during the assembly of the projector. Second, the voltage value of $5 \mathrm{~V}$ is stable and accurate and depends mainly on the quality of the linear regulator (131). This a good and easily accessible reference voltage that corresponds to the maximum input modulation signal that may be applied to the modulation input (132) of the driver. When this $5 \mathrm{~V}$ voltage is used as a modulation input for all three color channels simultaneously, a factory-calibrated white beam of high quality can be created by adjusting the three voltage dividers (133), which will be the default maximum power setting of the laser projector. Later, when customers use the laser projector with other input modulation voltage sources, the color balance can be additionally adjusted by means of the potentiometers mounted on the control panel of the projector. The connectors (132) for these potentiometers can be seen next to the connectors for the analog input modulation signals in Fig. 4.

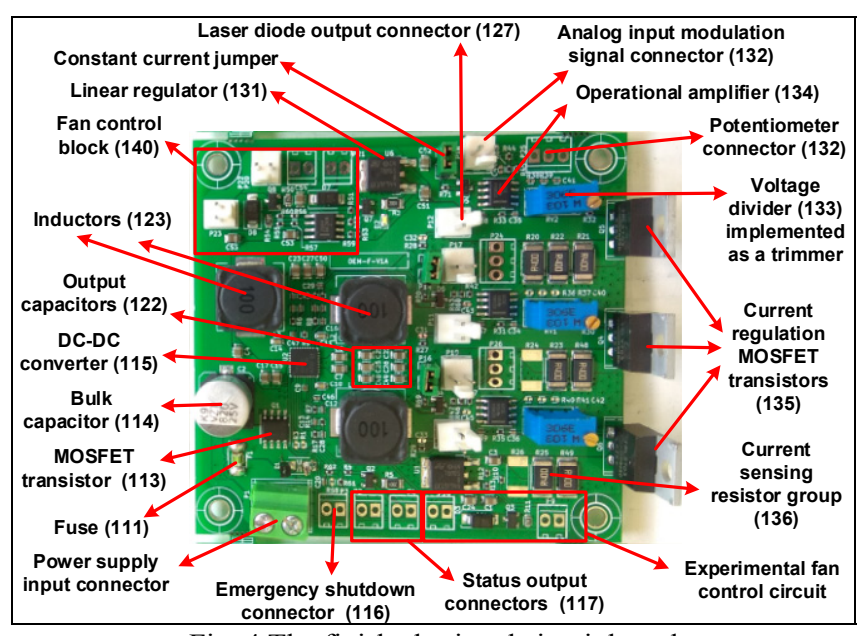

Fig. 4 The finished printed circuit board

The operational amplifiers (134) do the actual control of the current that passes through the MOSFET transistors (135) and the laser diodes connected to the output connectors (127). The operational amplifier and the corresponding output connector for each channel are located near each other (Fig. 4). The current sensing resisting group (136) is located between the MOSFET transistor (135) and the output connector (127) for each channel (Fig. 4). It may consist of two or three resistors of values less than $1 \Omega$ that are part of the feedback circuit of the operational amplifier (134). At the moment, we use three $0.2 \Omega$ resistors for the blue channel and two $0.2 \Omega$ resistors for the red and green channels in order to minimize the energy wasted in form of heat.

The DC-DC converter (115) and the other elements needed for the provision of power supply voltage to the three color channels are located on the left side of the PCB (Fig. 4). Among these elements are three inductors (123) and multiple output capacitors (122) in 0805 housings that stabilize the output voltage for each channel. At the input of the DC-DC converter (115), a large bulk capacitor (114) is connected, which stabilizes the input voltage. The MOSFET transistor (113) and the fuse (111) located next to the bulk capacitor
(114) provide protection against voltage polarity reversal or overcurrent at the input. The power supply input connector is located at the lower left corner of the PCB.

Near the bottom edge of the board in Fig. 4, an emergency shutdown connector (116) is placed. This connector can be used by an external microcontroller to pass an emergency shutdown signal to the laser diode driver, which will switch the DC-DC converter (115) off. The two status output connectors (117) may be used to provide a status feedback to the external microcontroller or to control a status LED indicating whether the DC-DC converter (115) is operational or not. Last but not least, we have incorporated an additional experimental fan control circuit near the bottom right corner of the PCB (Fig. 4), which uses the power failure detection feature of the DC-DC converter (115) to control a cooling fan.

The fan control block (140) is located at the top left corner of the PCB. It contains a dual operational amplifier (e.g. LM2904), two MOSFET transistors for switching the fans on or off, two freewheeling diodes, connectors for the thermistors and the fans and several capacitors and resistors to set the temperature thresholds for fan activation.

\section{EXPERIMENTAL RESULTS}

The three-channel laser diode driver exhibited very good response to the signals passed to its analog modulation inputs. For our applications, we target frequencies around $50 \mathrm{kHz}$ to $100 \mathrm{kHz}$ and our measurements show that we achieved good representation of square-waves at the output at these frequencies (Fig. 7 and Fig. 8). The driver also mapped the changes in the input modulation voltage to changes in the output current with a high degree of linearity. This is important for the good color representation of the projected animation.

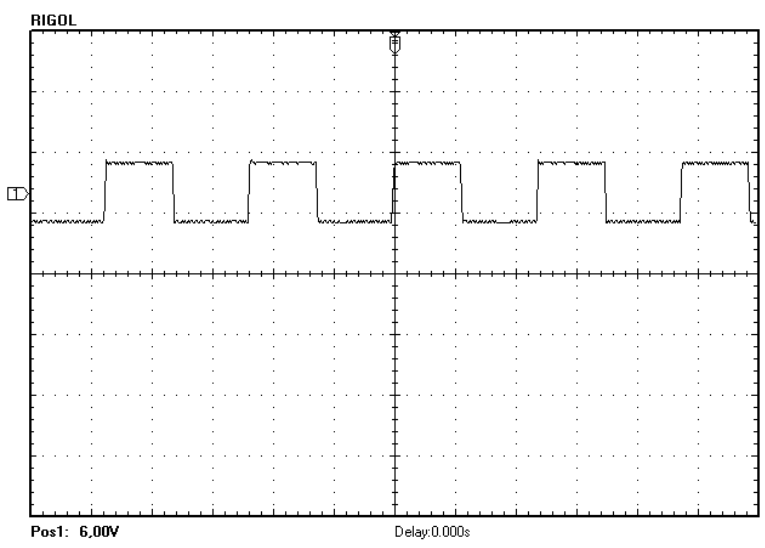

Fig. 5 Square-wave input modulation signal, $415 \mathrm{kHz}$

The square-wave modulation is one of the toughest cases for drivers of this class that are intended to drive between $1 \mathrm{~A}$ and $3 \mathrm{~A}$ of current and, usually, most frequency specifications are given for sinusoidal signals at the inputs. As multimedia laser projectors frequently need immediate "blanking" (switching off) of the laser beams during animations, square 
waves frequently occur at the modulation inputs and their quick processing is important for the projection of highquality laser animations.

As part of our test setup, we used a $5 \mathrm{~V}$ square-wave signal generated directly by an ATmega328p microcontroller operating at $5 \mathrm{~V}$ and equipped with an external $16 \mathrm{MHz}$ quartz crystal. As a power supply for the ATmega328p, we used one of the USB ports of the notebook used to program the microcontroller. A digital output pin of the ATmega328p was connected directly to the modulation input of one of the channels of the driver. A waveform of the square-wave input modulation signal at $415 \mathrm{kHz}$ is shown in Fig. 5.

The test setup also included a Meanwell GST60A12-P1J power supply connected to the power supply input connector of the driver (see Fig. 4). This power supply provides up to $5 \mathrm{~A}$ of current at $12 \mathrm{~V}$ and has a load regulation of $3 \%$ according to its datasheet. As measurement equipment, we used a Rigol DS1102D oscilloscope with a bandwidth of $100 \mathrm{MHz}$. The waveforms shown in Figs. 5-10 were generated by the Rigol software "Ultrascope".

The test procedure was as follows. First, we programmed the ATmega328p microcontroller to generate a square-wave signal of a predefined frequency and let it run while being connected to the laser driver. At the output of the laser driver, we connected a series (string) of five Schottky diodes, each with a forward voltage of about $0.45 \mathrm{~V}$, that emulated a red laser diode. We targeted specifically the HL63193MG red laser diode with a forward voltage of $2.2 \mathrm{~V}$ but did not want to damage it in the first tests if anything went wrong. The output results represent voltages that were measured in AC coupling mode by the oscilloscope across the current sensing resistor group (136). We chose this setup in order to be able to map the changes in the measured voltage directly to changes in the output current, which correspond to changes in the laser diode intensity.

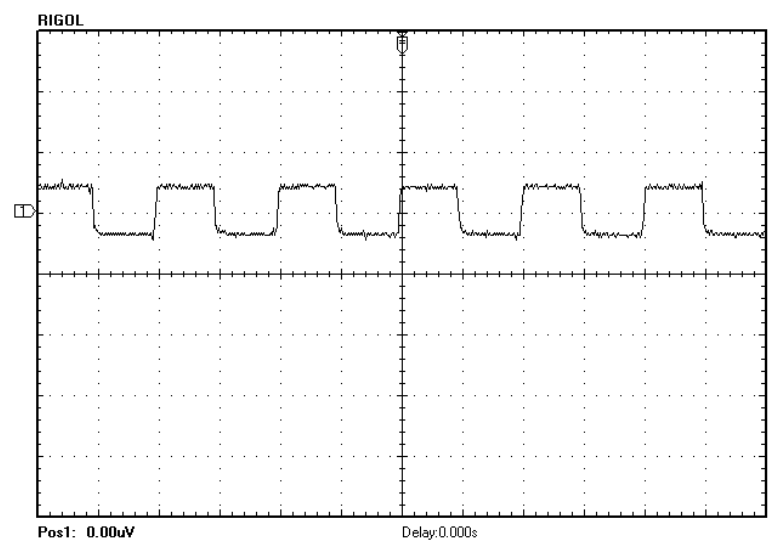

Fig. 6 Results of a square-wave modulation, $10 \mathrm{kHz}$

Our test results show that modulation frequencies around $10 \mathrm{kHz}$ are mirrored very well (Fig. 6). In Figs. $6-10$, the vertical axis resolution is $50 \mathrm{mV}$ per unit, while the horizontal axis resolution is respectively $50 \mu$ s per unit (Fig. 6), $10 \mu$ s per unit (Fig. 7), $5 \mu$ s per unit (Fig. 8), $2 \mu$ s per unit (Fig. 9) and $1 \mu$ s per unit (Fig. 10).

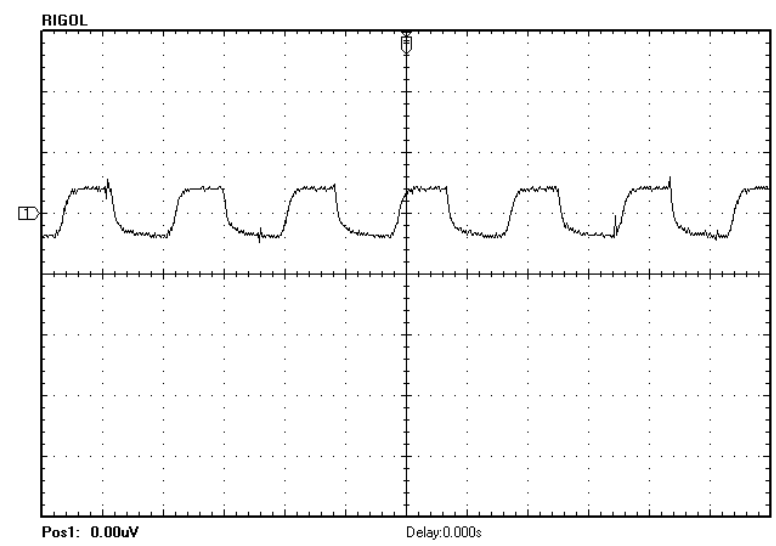

Fig. 7 Results of a square-wave modulation, $54 \mathrm{kHz}$

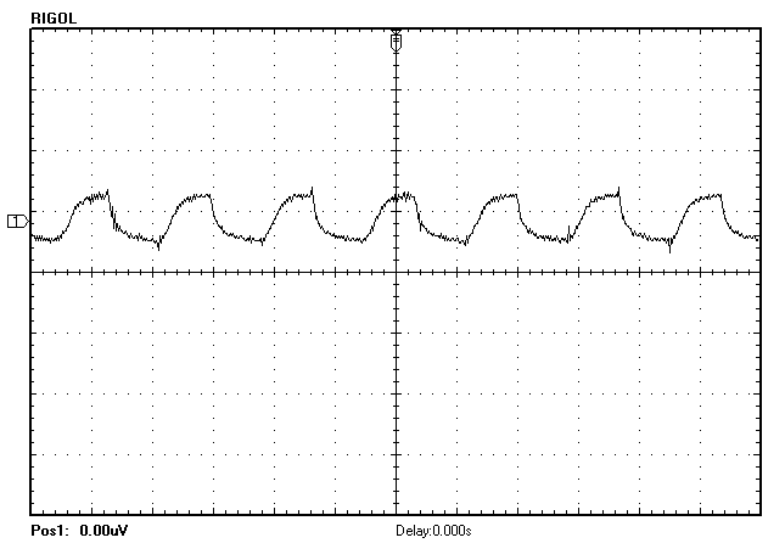

Fig. 8 Results of a square-wave modulation, $120 \mathrm{kHz}$

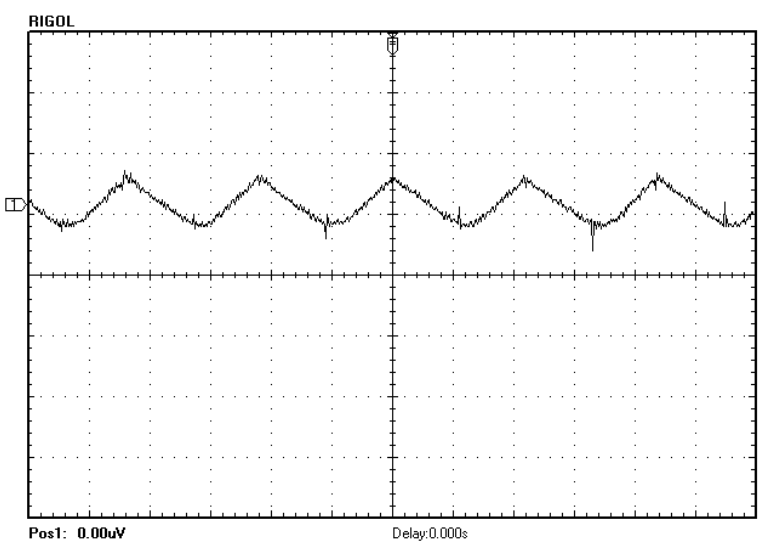

Fig. 9 Results of a square-wave modulation, $225 \mathrm{kHz}$

The good output representation of the input modulation signal continues at frequencies around $50 \mathrm{kHz}$ (Fig. 7), but at this stage the rising and falling edges of the output current are not as sharp as they could be, ideally. As modulation frequencies of $100 \mathrm{kHz}$ are reached, the waveform is still recognizable but it gradually begins morphing into a 
sinusoidal/triangular shape (Fig. 8).

This is mainly due to the relatively powerful but inexpensive MOSFETS (e.g. IRL2703PBF) that we use to transfer currents of up to several amperes to the laser diode strings. The sinusoidal/triangular shape becomes more pronounced at $225 \mathrm{kHz}$ (Fig. 9) and at $415 \mathrm{kHz}$ (Fig. 10). After replacing the string of five Schottky diodes with the real HL63193MG laser diode, we obtained similar results.

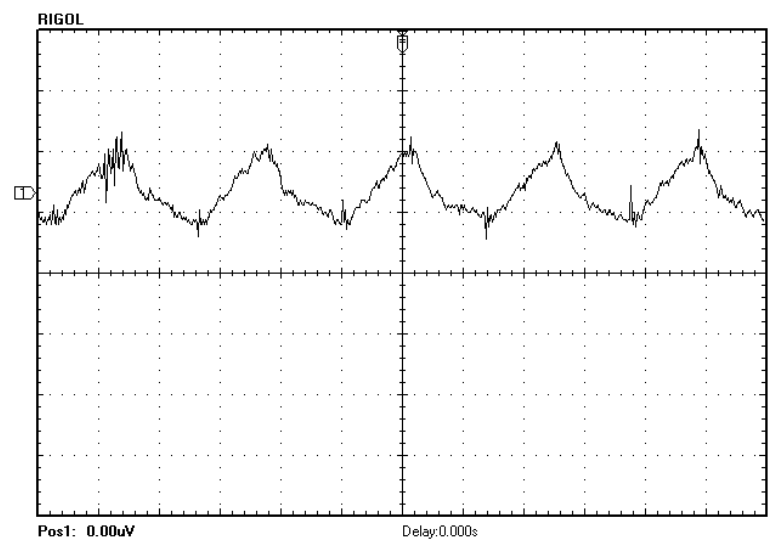

Fig. 10 Results of a square-wave modulation, $415 \mathrm{kHz}$

The modulation of a square wave, in contrast to sinusoidal waves, is one of the most challenging modes of operation for this application and our results were achieved without optimizations of the feedback circuit to improve the response of the operational amplifier (134). Our future efforts will be directed towards matching the feedback to the characteristics of the operational amplifier and the specific transistors that we use. We also plan to change the transistors to more expensive models with less capacitance so that steeper turn on/off times are achieved and we expect an improvement in the waveforms and the maximum supported frequencies.

The heat dissipation of the transistors was unproblematic when the respective outputs of DC-DC converter were adjusted to about $1 \mathrm{~V}$ above the total forward voltage of the respective laser diode strings (e.g. 3.2V for one HL63193MG laser diode and $5.6 \mathrm{~V}$ for one NDG7475 laser diode). Operating temperatures under $40^{\circ} \mathrm{C}$ were the norm, with an attached heatsink of dimensions $75 \times 50 \mathrm{~mm}$ that was common for all transistors. The DC-DC converter itself got warmer up to $60^{\circ} \mathrm{C}$ when all three channels were utilized so we decided to attach a heatsink with dimensions equal to the board size $(75 \times 79 \mathrm{~mm})$ to the underside of the board. For the thermal contact, we used a thermal pad with a thickness of about $3 \mathrm{~mm}$. For the tests, we used the aforementioned regulated power supply at $12 \mathrm{~V}$ but we have the option of increasing the power supply voltage up to $18 \mathrm{~V}$ if we need larger laser diode strings in the future. The driver was also tested for long-term operation. After a duration of several hours, it showed stable working temperatures and stable modulation characteristics.

\section{CONCLUSION}

The laser diode driver proposed in this paper controls the laser beam intensity of red, green and blue laser diodes that are part of a multimedia laser projector system. Among the benefits of the driver are its compact size, high efficiency and the very sensitive user-adjustable analog modulation inputs. Besides its main functionality, the driver also performs some auxiliary functions such as fan control, emergency shutdown of the laser diode strings and operational status indication. Furthermore, the driver design is economical and it achieves the analog control of the laser beam intensity without the use of rare or restricted components.

One of our contributions is the design of the functional blocks of the driver and the corresponding functional groups, which include a three-channel DC-DC converter used to provide power supply to three separate laser diode channels, whose current is controlled by analog voltage modulation. Another contribution is the physical implementation in the form of a printed circuit board. In addition, we did some measurements of the performance and evaluated the results. In general, we are very satisfied and our future work will encompass the fine-tuning of the current regulation feedback to guarantee a high-quality mixed-color beam for any input signal combinations and improved timing characteristics.

\section{ACKNOWLEDGMENT}

This research is supported by the National Scientific Program "Information and Communication Technologies for a Single Digital Market in Science, Education and Security (ICTinSES)", financed by the Ministry of Education and Science.

\section{REFERENCES}

[1] C. Canal, A. Laugustin, A. Kohl, and O. Rabot. (2017). Disruptive laser diode source for embedded LIDAR sensors, Proc. SPIE LASE, 2017, San Francisco, California, United States, 2017, pp. 10086 - 10086 - 7. DOI: $10.1117 / 12.2250197$.

[2] D. Lee, and J. Moore. (2018). Circuit and Method of Operating a Laser Diode. US Patent US20180145482A1. URL: http://appft1.uspto.gov/netacgi/nph-

Parser?Sect $1=$ PTO $1 \&$ Sect $2=$ HITOFF $\& d=$ PG01 $\& \mathrm{p}=1 \& u=/$ netahtml $/ \mathrm{PT}$ $\mathrm{O} /$ srchnum.html\& $\mathrm{r}=1 \& \mathrm{f}=\mathrm{G} \& \mathrm{l}=50 \& \mathrm{~s} 1=20180145482 . \mathrm{PGNR}$.

[3] J. Glaser. (2018). High Power Nanosecond Pulse Laser Driver Using an GaN FET, PCIM Europe 2018; International Exhibition and Conference for Power Electronics, Intelligent Motion, Renewable Energy and Energy Management, Nuremberg, Germany, 2018, pp. 1-8. URL: $\mathrm{http}$ ://ieeexplore.ieee.org/stamp/stamp.jsp?tp=\&arnumber=8402911\&isn umber $=8402799$.

[4] J. Glaser. (2018). Kilowatt Laser Driver with 120 A, sub-10 nanosecond pulses in $<3 \mathrm{~cm}^{2}$ using an GaN FET, PCIM Asia 2018; International Exhibition and Conference for Power Electronics, Intelligent Motion, Renewable Energy and Energy Management, Shanghai, China, 2018, pp. 1-6. URL: http://ieeexplore.iee.org/stamp/stamp.jsp?tp=\&arnumber=8470507\&isn umber $=8470501$.

[5] E. Fulkerson, R. Lanning, and S. Telford. (2014). Compact high current, high efficiency laser diode driver. US Patent US9972969B2. URL: http://patft.uspto.gov/netacgi/nphParser?Sect2=PTO $1 \&$ Sect $2=$ HITOFF $\& \mathrm{p}=1 \& \mathrm{u}=/$ netahtml $/ \mathrm{PTO} /$ searchbool.html\&r=1\&f=G\&l=50\&d=PALL\&RefSrch=yes\&Query=PN/99729 69. 
[6] M. Kabel. (2016). High Speed Laser Diode Driver. DOI: 10.13140/RG.2.2.30013.15841.

[7] M. Thompson, and M. Schlecht. (1997). High power laser diode driver based on power converter technology, IEEE Transactions on Power Electronics, vol. 12 , no. 1, pp. 46-52, Jan. 1997, DOI $10.1109 / 63.554168$.

[8] A. Stylogiannis, L. Prade, A. Buehler, J. Aguirre, G. Sergiadis, and V. Ntziachristos. (2018). Continuous wave laser diodes enable fast optoacoustic imaging, Photoacoustics, Volume 9, 2018, Pages 31-38, ISSN 2213-5979, DOI: https://doi.org/10.1016/j.pacs.2017.12.002.

[9] N. Denev, I. Iliev, and S. Gocheva-Ilieva. (2013). Second-Degree Polynomial Model of Laser Generation for a $\mathrm{CuBr}$ Laser, WSEAS Transactions on Circuits and Systems, Issue 4, Volume 12, April 2013, pp. 129-139, E-ISSN: 2224-266X.

[10] S.-Y. Cho. (2019). Locating Laser Sensors for Projector Touch Screens using Trigonometric Methods, WSEAS Transactions on Mathematics, Volume 18, 2019, pp. 147-152, ISSN / E-ISSN: 1109-2769 / 2224-2880.

[11] S. Kristiyana, and D. Dwanurendra. (2020). Laser Guiding of Three Phase Tesla Coil High Voltage Discharges, WSEAS Transactions on Electronics, Volume 10, 2020, Art. \#7, pp. 54-59, ISSN / E-ISSN: 11099445 / 2415-1513, DOI: 10.37394/232017.2020.11.7.

[12] M. Anarghya, S. Nitish, R. Yatheesha, and B. Gurumurthy, (2016). Investigation of $\mathrm{CO} 2$ Laser Drilled Micro Holes for Heat Affected Zone and Structural Integrity in CFRP Composites, International Journal of Materials, Volume 3, 2016, pp. 33-43, ISSN: 2313-0555.

[13] L. Sykorova, and O. Suba. (2011). The Transient Temperature Field Simulation of Polymeric Materials During Laser Machining, International Journal of Mechanics, Issue 1, Volume 5, 2011, pp., 234241, ISSN: 1998-4448.

[14] H. Vašková. (2011). A powerful tool for material identification: Raman spectroscopy, International Journal of Mathematical Models and Methods in Applied Sciences, Issue 1, Volume 5, 2011, pp. 1205-1212, ISSN: 1998-0140.

[15] E. Paunova-Hubenova, V. Terzieva, S. Dimitrov, and Y. Boneva (2018). Integration of Game-Based Teaching in Bulgarian Schools State of Art. Proc. of 12th European Conference on Game-based Learning ECGBL 2018, October 4-5 2018, Sophia Antipolis, France, pp. 516-525, ISSN 2049-0992.

[16] T. Savov, V. Terzieva, and K. Todorova. (2018). Computer Vision and Internet of Things: Attention System in Educational Context. Proc. of the 19th International Conference on Computer Systems and Technologies (CompSysTech'18), Boris Rachev and Angel Smrikarov (Eds.). ACM, New York, NY, USA, 13-14 September 2018, Ruse, Bulgaria, pp. 171-177, DOI: $10.1145 / 3274005.3274014$.

[17] Y. Boneva. (2018). Optimization of Car Traffic Flow on Intersections Regulated by Traffic Lights Through the Simulation Environment AIMSUN. Academic journal Mechanics Transport Communications, ISSN 1312-3823 (print), ISSN 2367-6620 (online), Vol. 16, issue 2, 2018, Todor Kableshkov University of Transport, Bulgaria, pp. I-1-I-9, URL: https://mtc-aj.com/library/1663.pdf.

[18] A. Alexandrov, and V. Monov. (2017). Method for indoor localization optimization of AoA based mobile devices. Proc. of 12th Annual Meeting of the Bulgarian Section of SIAM BGSIAM'17, December 2022, 2017, Sofia, Bulgaria, 2017, ISSN:1313-3357.

[19] A. Alexandrov, and V. Monov. (2018). Method for Adaptive Node Clustering in AD HOC Wireless Sensor Networks. Vishnevskiy V., Kozyrev D. (eds) Distributed Computer and Communication Networks. DCCN 2018. Communications in Computer and Information Science, vol. 919, Springer, Cham, ISBN: 978-3-319-99447-5, DOI 10.1007/978-3-319-99447-5 22

[20] A. Alexandrov, and V. Monov. (2014). ZigBee smart sensor system with distributed data processing. Proc. of the 7-th IEEE Conference Intelligent Systems, Warsaw Poland, Vol. 2, pp. 259-268, September 2428, 2014., Advances in Intelligent Systems and Computing, Springer, vol. 323, ISBN 978-3-319-11309-8, DOI: 10.1007/978-3-319-11310423.

[21] V. Ivanova, D. Bachvarov, and A. Boneva. (2018). An Advanced Robot System for Diagnostic and Therapeutics Tasks with Application in Laparoscopic Surgery. Journal of Computer Engineering \& Information Technology, Vol. 7, Issue 2, ISSN: 2324-9307 (Online), DOI: 10.4172/2324-9307.1000198, SciTechnol, 2018, London, United Kingdom, pp. 1-9.
[22] S. Ilchev, R. Andreev, and Z. Ilcheva. (2019). Ultra-Compact Laser Diode Driver for the Control of Positioning Laser Units in Industrial Machinery. IFAC Papers Online, Edited by Larry Stapleton, Peter Kopacek, Andon Topalov, Vol. 52, Issue 25, 2019, ISSN 2405-8963, pp. 435-440 and Proc. of 19th IFAC Conference on Technology, Culture and International Stability (TECIS 2019), 26-28 Sep. 2019, Sozopol, Bulgaria, DOI: 10.1016/j.ifacol.2019.12.577.

Svetozar Ilchev received his Ph.D. degree from the Bulgarian Academy of Sciences in 2014. His M.Sc. degree in Information Engineering and Management was completed in 2009 at the Karlsruhe Institute of Technology. $\mathrm{He}$ is interested in the development of innovative embedded systems and privacy-conscious IT solutions based on new hardware products and web applications. The main objective of his research is to meet the contemporary needs of human users for interaction, networking and advertising while respecting their privacy and personal data. Dr. Ilchev is currently working as a research associate at the Institute of Information and Communication Technologies, Bulgarian Academy of Sciences.

Rumen Andreev (b. 1955, Sofia, Bulgaria) is an Associate Professor since 2010 at the Institute of Information and Communication Technologies, Bulgarian Academy of Sciences. He received his Ph.D. degree in 1988 in the field of Computer Aided Design in Machinery Construction from the Higher Institute of Machine and Electrical Engineering (Technical University), Sofia, Bulgaria. Currently, Dr. Andreev is head of the department of Communication Systems and Services at the Institute of Information and Communication Technologies, Bulgarian Academy of Sciences. His main research areas include complex systems (ecosystems), communication systems and networks and e-learning. He has been a participant and a manager of more than 40 research projects and he is the author and co-author of a significant number of international and national scientific publications.

Zlatoliliya Ilcheva received her Ph.D. degree from the Bulgarian Academy of Sciences in 1980. She received her M.Sc. Degree in Automatics and Telemechanics from the Technical University - Sofia in 1975. Her research interests include Man-Machine Control Systems, Pattern Recognition, Image Processing, Image Compression and Multimedia Data Hiding. She is the author of a large number of publications in the aforementioned research areas. She has participated in many research and industrial projects financed by both national and international partners and programs. Dr. Ilcheva is currently working as an Associate Professor at the Institute of Information and Communication Technologies, Bulgarian Academy of Sciences.

Ekaterina Otsetova-Dudin received her Ph.D. degree from the University of Ruse "Angel Kanchev" in 2015. She received her M.Sc. Degree in Electronics and Automatics from the Technical University - Sofia in 1992. Her research interests include telecommunications, computer networks and e-learning. She has participated in several research projects pertaining to telecommunications and e-learning and has multiple research publications on these topics. Dr. Otsetova-Dudin is currently working as a research associate at the University of Telecommunications and Posts in Sofia, Bulgaria.

\section{Creative Commons Attribution License 4.0 (Attribution 4.0 International, CC BY 4.0)}

This article is published under the terms of the Creative Commons Attribution License 4.0

https://creativecommons.org/licenses/by/4.0/deed.en_US 